also contained microbes which can oxidize manganese, for supplements of manganous sulphate produced typical signs of deposition. Only the very low level of manganous salts prevented noticeable accretions in the pipelines.

of the various bacteria and fungi implicated in the pipeline depositions, a stalked budding bacterium, Hyphomicrobium, predominated. On artificial media this bacterium formed dense brown colonies surrounded by haloes caused by buds growing out from the edge of the manganese particle. The stalks were frequently branched and anastomosed and in this respect resembled the manganese-oxidizing bacterium Pedomicrobium described some years ago by Aristovskaya. Tyler and Marshall have carefully examined the morphology of these stalked bacteria (J. Bact., 93, 1132; 1967) and, on the basis of extreme pleomorphy, conclude that the genus Pedomicrobium is invalid and should be regarded as a form of Hyphomicrobium.

The mechanism of manganese deposition has not been clarified, but the direct oxidation of soluble manganous salts to an insoluble state is an obvious possibility. Alternatively, micro-organisms may assimilate organic parts from organo-manganese chelates in the lake water, particularly with water from Lake King William, thence promoting the accumulation of residual manganese. Tyler and Marshall (Arch. fur Mikrobiol., 56, 344; 1967) regard the pipeline habitat as an extended continuous culture vessel through which lake water feeds at a relatively constant rate. Because of the high flow rate and low level of nutrient, manganese precipitation in the water itself is negligible. On the other hand, organisms able to attach themselves to the pipeline may avail themselves of the continuous supply of lake water medium, dilute though it may be. A selection process of this kind can be compared with the condition of "take-over" in continuous cultures of Escherichia coli tyr $^{-}$reported by Munson and Bridges. They found that prototrophic revertants have an increased capacity for adhering to the vessel walls and rapidly dominate the system. The way in which hyphomicrobia attach to the pipelines needs further study, however. A direct electrostatic mechanism is unlikely because the pipes and the bacteria are both negatively charged. The pipe surface is, however, likely to attract various cations present in the lake water, and these may provide both electrostatic attraction sites and critical concentrations of nutrients for the bacteria.

One intriguing possibility is that the morphology of these bacteria may be highly significant to their mode of existence. The growth of bacillary forms would be severely limited by self-produced encrustations of manganese. By contrast, hyphomicrobia produce buds at the ends of long stalks when their older cells become isolated from the nutrients in this way, and so can evade the restrictive environment of the deposit. This pattern of development has been verified both in pipeline deposits and in pure cultures actively precipitating manganese.

\section{Specificity in RNA Synthesis}

from a Correspondent in Molecular Biology

IF to travel hopefully is indeed better than to arrive, then the state of progress on RNA synthesis in the cell must give some satisfaction. At the same time many interesting experimental observations are emerging which must in time begin to form a coherent pattern. An enzyme which has been of more obvious value to the molecular biologist than to the cell is polynucleo. tide phosphorylase. It is used everywhere for the preparation of synthetic polyribonucleotides, but its purpose in vivo is unknown, though this is presumed to be degradative rather than synthetic.

Some interesting if only partially interpretable results now come from Grunberg-Manago's laboratory (Harvey et al., European J. Biochem., 1, 327; 1967). It appears that the enzyme is very sensitive to urea, which affects it in two quite different ways: at concentrations of no more than $3 M$ it produces irreversible denaturation but, short of denaturation, it strongly (but reversibly) inhibits the polymerization reaction. All oligo- and poly-ribonucleotides give protection against the denaturation, but reversal of the inhibition is much more specific, for it requires that the oligonucleotide terminate in a $3^{\prime}$-hydroxyl group and not a $3^{\prime}$-phosphate. Neither are polymers of high molecular weight effective. The authors interpret their results in terms of two different modes of binding. It is interesting that the same concentrations of urea have only a minimal effect on the reverse (depolymerization) reaction. This supports the authors' contention that inhibition of polymerization occurs at initiation, rather than in propagation: an oligonucleotide with a 3 -hydroxyl terminus is known to function as an initiator (primer), and so eliminates the usual initiation lag, whereas a $3^{\prime}$-phosphate group prevents this.

A similar distinction between initiation and propagation has been noted in the function of DNA-dependent RNA polymerase, an enzyme whose biological relevance is in no doubt. It has been a central problem to establish what determines the point in the DNA chain at which the enzyme begins to transeribe. Cohen et al. (J. Mol. Biol., 26, 19; 1967) have now found, using fragments of DNA from the bacteriophage $\lambda$, that regions of high template activity exist in the segment known to be rich in $\mathrm{AT}$, and that transcription occurs there throughout the reaction. This specificity is destroyed when the DNA is denatured. It has been known for some time, however, that binding to the template is not necessarily followed by transcription, and indeed it was found that both halves of $\lambda$ DNA bound enzyme and initiated synthesis of RNA, but that it was the actual transeription process which was inefficient on the GC-rich part.

This difference between binding and propagation has also been studied by Hirschbein et al. (European $J$. Biochem., 1, 135; 1967). They have followed inhibition by synthetic polyribonucleotides, which bind to the enzyme and can also in principle function as templates in place of DNA. Thus poly-C can be transeribed, but it turns out to be a relatively poor inhibitor of DNA transcription. Poly-G and poly-I, on the other hand, have no template activity whatever, but bind so strongly to the enzyme that they can be used as trapping agents. Two-stranded poly- $(G+C)$ binds little, and is not transcribed. Differential binding studies with this and with poly- $(\mathrm{A}+\mathrm{U})$ show conclusively that the enzyme will recognize bases in a two-stranded polynucleotide, and also that it will not necessarily catalyse transcription at the points at which it binds most readily. 